\title{
APPROXIMATION BY POLYNOMIALS SUBORDINATE TO A UNIVALENT FUNCTION $\left({ }^{1}\right)$
}

\author{
BY
}

THOMAS H. MACGREGOR

This paper is concerned with approximating a function $f(z)$ analytic and univalent in the unit disk $E=\{z:|z|<1\}$ by polynomials which are also univalent in $E$. We are interested in such approximations where exactly one polynomial of each degree $n, n \geqq 1$, is used and such that the polynomials are monotonically subordinate to each other.

Recall that $f(z)$ is called subordinate to $g(z)$ in $E$ if both functions are analytic in $E$ and if there exists a function $\varphi(z)$ analytic in $E$ and satisfying $|\varphi(z)|<1$, $\varphi(0)=0$ such that $f(z)=g(\varphi(z))$. The existence of such a function $\varphi(z)$ is implied by the conditions that $g(z)$ is univalent in $E, f(0)=g(0)$ and $f(E) \subset g(E)$. If $f(z)$ is subordinate to $g(z)$ in $E$ we will write $f(z) \subset g(z)$ in $E$.

Theorem 1 asserts that if $f(z)$ is analytic and univalent in $E$ then there exists a sequence $\left\{p_{n}(z)\right\}, n=1,2, \ldots$, such that $p_{n}(z)$ is a polynomial of degree $n$ which is univalent in $E$,

$$
p_{1}(z) \subset p_{2}(z) \subset p_{3}(z) \subset \cdots
$$

and $p_{n}(z) \rightarrow f(z)$ as $n \rightarrow \infty$. This convergence is uniform in each compact subset of $E$. The idea of proving this is to appropriately relate $f(z)$ to the partial sums $s_{n}(z)$ of the power series for $f(z)$. Although $s_{n}(z)$ are not, in general, univalent in $E$ (they are in $|z|<\frac{1}{4}[12]$ ) they are univalent in $|z|<r, 0<r<1$, for all large $n$ once $r$ is given. This eventually leads to a chain of univalent polynomials

$$
p_{n_{1}}(z) \subset p_{n_{2}}(z) \subset p_{n_{3}}(z) \subset \cdots
$$

such that $p_{n_{j}}(z)$ has degree $n_{j}, n_{1}<n_{2}<n_{3}<\cdots$, and $p_{n_{j}}(z) \rightarrow f(z)$ as $j \rightarrow \infty$. These polynomials are so related that it is still possible to find polynomials of the remaining degrees which fill in the chain and such that $p_{n}(z) \rightarrow f(z)$.

This method is equally adaptable when $f(z)$ maps $E$ one-to-one onto a convex domain in order to produce a similar chain of convex, univalent polynomials. In fact, our argument may be applied to any one of a number of classes of univalent functions.

Received by the editors April 29, 1969.

( ${ }^{1}$ This research was partially supported by the National Science Foundation through the grant GP-8199. 
The possibility of such an approximation to convex mappings was implicitly raised by G. Pólya and I. J. Schoenberg in [10]. They considered analytic functions

$$
f(z)=\sum_{k=1}^{\infty} a_{k} z^{k}
$$

which map $E$ one-to-one onto a convex domain. For each integer $n, n \geqq 1$, the de la Vallée Poussin means of $f(z)$ are defined by

$$
V_{n}(z)=\frac{n}{n+1} a_{1} z+\frac{n(n-1)}{(n+1)(n+2)} a_{2} z^{2}+\cdots+\frac{n(n-1) \cdots 1}{(n+1)(n+2) \cdots(2 n)} a_{n} z^{n} .
$$

They showed that $V_{n}(z)$ is a convex, univalent function, $V_{n}(z) \subset f(z)$, and $V_{n}(z)$ $\rightarrow f(z)$ in $E$, and conjectured that

$$
V_{1}(z) \subset V_{2}(z) \subset V_{3}(z) \subset \cdots
$$

This conjecture remains open and its validity would yield a somewhat more explicit proof of the existence of such a chain except that we also require $p_{n}(z)$ to have the exact degree $n$.

Disregarding the demand that $p_{n}(z)$ be a polynomial, it is easy to find a chain

$$
f_{1}(z) \subset f_{2}(z) \subset f_{3}(z) \subset \ldots
$$

such that $f_{n}(z) \rightarrow f(z)$ and we do not have $f_{n}(z)=f(z)$ for all $n$. One simply sets $f_{n}(z)=f\left(r_{n} z\right)$ where $\left\{r_{n}\right\}$ is an increasing sequence of real numbers in the interval $0<r<1$ such that $r_{n} \rightarrow 1$. A less trivial situation in the case $f(z)$ is univalent and convex in $E$ is obtained by considering the functions

$$
f(z, \alpha)=\frac{1}{\alpha} \int_{0}^{\alpha} f\left(z e^{i \theta}\right) d \theta
$$

If $\left\{\alpha_{n}\right\}$ is any sequence of real numbers in the interval $0<\alpha<2 \pi$ which monotonically converges to zero then $f\left(z, \alpha_{n}\right)$ is univalent and convex, $f\left(z, \alpha_{1}\right) \subset f\left(z, \alpha_{2}\right)$ $\subset f\left(z, \alpha_{3}\right) \subset \ldots$ and $f\left(z, \alpha_{n}\right) \rightarrow f(z)$ in $E$ [6].

Interest in (continuous as well as discrete) chains of subordinate functions has occurred elsewhere and we would like to point out the recent papers by C. Pommerenke [7], [8], [9] as well as [1] by A. Bielecki and Z. Lewandowski.

The earliest consideration of this kind presumably is due to $\mathrm{C}$. Carathéodory in his idea of kernels.

LEMMA 1. Let $f(z)=\sum_{k=0}^{\infty} a_{k} z^{k}$ be analytic in $E$ and set $s_{n}(z)=\sum_{k=0}^{n} a_{k} z^{k}$.

Let $\left\{n_{j}\right\}$ be a strictly increasing sequence of positive integers and let $\left\{r_{j}\right\}$ be a sequence of real numbers such that $0<r_{j}<1$ and $r_{j} \rightarrow 1$ as $j \rightarrow \infty$. Then $s_{n_{j}}\left(r_{j} z\right) \rightarrow f(z)$ uniformly in each disk $|z| \leqq r, 0<r<1$. 
Proof. Suppose that $|z| \leqq r$ and $0<r<1$.

$$
\begin{aligned}
\left|f(z)-s_{n_{j}}\left(r_{j} z\right)\right| & =\left|\sum_{k=0}^{n_{j}} a_{k}\left(1-r_{j}^{k}\right) z^{k}+\sum_{k=n_{j}+1}^{\infty} a_{k} z^{k}\right| \\
& \leqq\left(1-r_{j}\right) \sum_{k=0}^{n_{j}} k\left|a_{k}\right| r^{k}+\sum_{k=n_{j}+1}^{\infty}\left|a_{k}\right| r^{k} \\
& \leqq\left(1-r_{j}\right) \sum_{k=0}^{\infty} k\left|a_{k}\right| r^{k}+\sum_{k=n_{j}+1}^{\infty}\left|a_{k}\right| r^{k}
\end{aligned}
$$

Given a positive number $\varepsilon$ there is an integer $N$ such that $\sum_{k=n+1}^{\infty}\left|a_{k}\right| r^{k}<\varepsilon / 2$ if $n \geqq N$. Since $n_{j} \geqq j$ this implies that for $j \geqq N, \sum_{k=n_{j}+1}^{\infty}\left|a_{k}\right| r^{k}<\varepsilon / 2$. As $r_{j} \rightarrow 1$ there is an integer $N^{\prime}$ such that if $j \geqq N^{\prime}$ then

$$
\left(1-r_{j}\right) \sum_{k=0}^{\infty} k\left|a_{k}\right| r^{k}<\frac{\varepsilon}{2}
$$

Choosing $N^{\prime \prime}=\max \left(N, N^{\prime}\right)$ we see that $\left|f(z)-s_{n_{j}}\left(r_{j} z\right)\right|<\varepsilon$ for $j \geqq N^{\prime \prime}$ and this proves the lemma.

THEOREM 1. Let $f(z)$ be analytic and univalent in E. There exists a sequence of polynomials $\left\{p_{n}(z)\right\}, n=1,2,3, \ldots$, where $p_{n}(z)$ has degree $n$ and is univalent in $E$, such that

$$
p_{1}(z) \subset p_{2}(z) \subset p_{3}(z) \subset \ldots
$$

in $E$ and $p_{n}(z) \rightarrow f(z)$ in $E$. The convergence is uniform in compact subsets of $E$.

Proof. Let the power series for $f(z)$ be $f(z)=\sum_{k=0}^{\infty} a_{k} z^{k}$ and let $s_{n}(z)=\sum_{k=0}^{n} a_{k} z^{k}$. Then $s_{n}(z) \rightarrow f(z)$ and $s_{n}^{\prime}(z) \rightarrow f^{\prime}(z)$ as $n \rightarrow \infty$ uniformly in each disk $|z| \leqq r$, where $0<r<1$.

Let us introduce the notation:

$$
\begin{aligned}
\Delta_{g}(u, v) & =(g(u)-g(v)) /(u-v), & & \text { if } u \neq v, \\
& =g^{\prime}(u), & & \text { if } u=v,
\end{aligned}
$$

for each function $g(z)$ analytic in $E$. If $u \neq v,|u| \leqq r,|v| \leqq r, 0<r<1$, we may write

$$
g(u)-g(v)=\int_{v}^{u} g^{\prime}(z) d z
$$

where the path of integration is the line segment from $v$ to $u$. Since the points on this line segment satisfy $|z| \leqq r$, it is clear that

$$
\max _{|u| \leqq r,|v| \leqq r}\left|\Delta_{g}(u, v)\right|=\max _{|z| \leqq r}\left|g^{\prime}(z)\right| .
$$

Applying this to $g_{n}(z) \doteq f(z)-s_{n}(z)$ we conclude that $\Delta_{g_{n}}(u, v)$ is uniformly small for large $n$ if $|u| \leqq r,|v| \leqq r$, since $g_{n}^{\prime}(z)$ is uniformly small for large $n$ in $|z| \leqq r$.

Since $f(z)$ is univalent in $E, f^{\prime}(z)$ does not vanish, and, therefore,

$$
m_{f}(r)=\min _{|u| \leqq r,|v| \leqq r}\left|\Delta_{f}(u, v)\right|>0,
$$


where $0<r<1$. Because $\Delta_{s_{n}}(u, v)=\Delta_{f}(u, v)-\Delta_{g_{n}}(u, v)$ there exists an integer $N$ such that if $n \geqq N$ then $m_{s_{n}}(r) \geqq m_{f}(r) / 2$. In particular, this implies that $s_{n}(z)$ is univalent in $|z| \leqq r$ for all $n \geqq N$.

Let $D(r)$ denote the image of $|z|<r$ under $f(z)$ and let $D_{n}(r)$ denote the image of $|z|<r$ under $s_{n}(z)$, where $0<r \leqq 1$. Also, let $d(A, B)$ denote the distance between the sets $A$ and $B$ and let $\delta A$ denote the boundary of the set $A$. If we set $d(r)=$ $d(D(r), \delta D(1))$, where $0<r<1$, then $d(r)<\infty$ since $D(r)$ is bounded and $D(1)$ is not the whole plane. Also, $d(r)>0$ as $f(z)$ is an open mapping. There is an integer $N^{\prime}$ such that

$$
\left|f(z)-s_{n}(z)\right|<d(r) / 2
$$

for $|z| \leqq r, 0<r<1$, if $n \geqq N^{\prime}$. Consequently, if $n \geqq N^{\prime}$ then $d_{n}(r)=d\left(D_{n}(r), \delta D(1)\right)$ $>d(r) / 2$.

Let $r_{1}$ be any fixed number such that $0<r_{1}<1$ and choose an integer $n_{1} \geqq$ $\max \left(N, N^{\prime}\right)$, where $N=N\left(r_{1}\right), N^{\prime}=N^{\prime}\left(r_{1}\right)$ was obtained by the two previous arguments. This implies that $m_{s_{n_{1}}}\left(r_{1}\right) \geqq m_{f}\left(r_{1}\right) / 2$ and $d_{n_{1}}\left(r_{1}\right)>d\left(r_{1}\right) / 2$.

We continue our argument with the assumption that $f(z)$ is not a polynomial. Then the selection of $n_{1}$ can be made so that $s_{n_{1}}(z)$ has exactly the degree $n_{1}$. If $E_{n}(r)=f^{-1}\left(D_{n}(r)\right)$ then there is a number $\rho_{1}, 0<\rho_{1}<1$, such that $E_{n_{1}}\left(r_{1}\right)$ is contained in $|z| \leqq \rho_{1}$, as, otherwise, $d_{n_{1}}\left(r_{1}\right)=0$. Choose the number $r_{2}$ such that $\rho_{1}<r_{2}$ $<1$ and $r_{2}>\left(1+r_{1}\right) / 2$. Then $d\left(D_{n_{1}}\left(r_{1}\right), \delta D\left(r_{2}\right)\right)>0$ and, as always, $d\left(r_{2}\right)>0$.

Arguing as before we conclude that there is an integer $N=N\left(r_{2}\right)$ such that if $n \geqq N$ then $m_{s_{n}}\left(r_{2}\right) \geqq m_{f}\left(r_{2}\right) / 2$. Also there is an integer $N^{\prime}=N^{\prime}\left(r_{2}\right)$ such that if $n \geqq N^{\prime}$ then $\left|f(z)-s_{n}(z)\right|<d^{*} / 2$ for $|z| \leqq r_{2}$, where

$$
d^{*}=\min \left(d\left(r_{2}\right), d\left(D_{n_{1}}\left(r_{1}\right), \delta D\left(r_{2}\right)\right)\right) .
$$

This implies that $d_{n}\left(r_{2}\right)>d\left(r_{2}\right) / 2>0$ and

$$
d\left(D_{n_{1}}\left(r_{1}\right), \delta D_{n}\left(r_{2}\right)\right)>\frac{1}{2} d\left(D_{n_{1}}\left(r_{1}\right), \delta D\left(r_{2}\right)\right)>0
$$

for $n \geqq N^{\prime}$. The last condition and the facts that $D_{n_{1}}\left(r_{1}\right)$ is connected and $D_{n_{1}}\left(r_{1}\right)$ and $D_{n}\left(r_{2}\right)$ have a common point, namely $a_{0}$, implies that $D_{n_{1}}\left(r_{1}\right) \subset D_{n}\left(r_{2}\right)$.

If we set $N^{\prime \prime}=\max \left(N, N^{\prime}\right)$ then for $n \geqq N^{\prime \prime} m_{s_{n}}\left(r_{2}\right) \geqq\left(m_{f}\left(r_{2}\right) / 2\right), d\left(D_{n_{1}}\left(r_{1}\right), \delta D_{n}\left(r_{2}\right)\right)$ $>0$ and $d_{n}\left(r_{2}\right)>0$. The first two conditions and $s_{n}(0)=s_{n_{1}}(0)=a_{0}$ show that $s_{n}\left(r_{2} z\right)$ is univalent in $|z| \leqq 1$ and $s_{n_{1}}\left(r_{1} z\right) \subset s_{n}\left(r_{2} z\right)$ in $E$. Since $f(z)$ is not a polynomial there is such an integer $n=n_{2}$ so that $n_{2} \geqq N^{\prime \prime}, n_{2}>n$, and $s_{n_{2}}(z)$ has degree $n_{2}$. Moreover, since $d_{n_{2}}\left(r_{2}\right)>0$ this whole argument may be repeated.

Namely, we first consider the set $E_{n_{2}}\left(r_{2}\right)$ to show that there is a number $r_{3}$ such that $\left(1+r_{2}\right) / 2<r_{3}<1$ and $d\left(D_{n_{2}}\left(r_{2}\right), \delta D\left(r_{3}\right)\right)>0$.

We then find an integer $N$ associated with the condition $m_{s_{n}}\left(r_{3}\right) \geqq m_{f}\left(r_{3}\right) / 2$ and an integer $N^{\prime}$ associated with the inequality $\left|f(z)-s_{n}\right|<d^{*} / 2$ for $|z| \leqq r_{3}$, where

$$
d^{*}=\min \left(d\left(r_{3}\right), d\left(D_{n_{2}}\left(r_{2}\right), \delta D\left(r_{3}\right)\right)\right) \text {. }
$$


This leads to an integer $n_{3}$ so that $n_{3}>n_{2}, s_{n_{3}}(z)$ has degree $n_{3}, m_{s_{n_{3}}}\left(r_{3}\right) \geqq m_{f}\left(r_{3}\right) / 2$, $d\left(D_{n_{2}}\left(r_{2}\right), \delta D_{n_{3}}\left(r_{3}\right)\right)>0$, and $d_{n_{3}}\left(r_{3}\right)>0$. In particular, $s_{n_{3}}\left(r_{3} z\right)$ is univalent in $|z| \leqq 1$ and $s_{n_{2}}\left(r_{2} z\right) \subset s_{n_{3}}\left(r_{3} z\right)$ in $E$.

This argument, therefore, can be continued indefinitely and it yields the following conclusion. There is a sequence of real numbers $\left\{r_{j}\right\}$, a strictly increasing sequence of integers $\left\{n_{j}\right\}$ and a sequence of polynomials $\left\{p_{n_{j}}(z)\right\}$ such that $0<r_{j}<1$, $r_{j} \rightarrow 1$ and $p_{n_{j}}(z)=s_{n_{j}}\left(r_{j} z\right)$ are univalent in $|z| \leqq 1$. Moreover, $p_{n_{j}}(z)$ has degree $n_{j}$ and

in $E$. In addition,

$$
p_{n_{1}}(z) \subset p_{n_{2}}(z) \subset p_{n_{3}}(z) \subset \cdots
$$

and

$$
m_{p_{n_{j}}}(1) \geqq m_{f}\left(r_{j}\right) / 2
$$

$$
d\left(D_{n}\left(r_{j}\right), \delta D\left(r_{j+1}\right)\right)>0 .
$$

The fact that $r_{j} \rightarrow 1$ follows from the demand that

$$
\left(1+r_{j}\right) / 2<r_{j+1}<1 .
$$

We also note that because of Lemma $1 p_{n_{j}}(z) \rightarrow f(z)$ uniformly in every disk $|z| \leqq r, 0<r<1$.

The remaining part of our argument consists of interjecting appropriate polynomials between successive pairs in the chain

$$
p_{n_{1}}(z) \subset p_{n_{2}}(z) \subset p_{n_{3}}(z) \subset \cdots .
$$

Suppose that $p(z)$ and $q(z)$ are such a pair and have degrees $n$ and $k$, respectively, where $n<k$. Then $m_{p}(1)>0$ and therefore $p(z)$ is univalent in $|z|<R$ for some $R>1$. If $1<\rho<R$ and $P(z)=p(\rho z)$ then $m_{P}(1)>0$. Let

so that

$$
Q(z)=P(z)+b z^{n+1}
$$

$$
\left|\Delta_{Q}(u, v)\right| \geqq m_{P}(1)-(n+1)|b|
$$

in $|z| \leqq 1$. This implies that for all sufficiently small values of $b$

$$
m_{Q}(1) \geqq \frac{1}{2} m_{P}(1)
$$

so that, in particular, $Q(z)$ is univalent in $E$. If $A=p(E)$ and $B=q(E)$ then we have the condition $d(A, \delta B)>0$. Because of the kind of arguments made earlier in this proof it follows that for $\rho>1$ and sufficiently near 1 we can conclude that $p(E)$ $\subset P(E) \subset q(E)$ from the relations $d(p(E), \delta P(E))>0, d(P(E), \delta q(E))>0$, and $p(0)=P(0)=q(0)$.

As $Q(z) \rightarrow P(z)$ as $b \rightarrow 0$ uniformly in $|z| \leqq 1$ it is also possible to deduce that

$$
d(p(E), \delta Q(E))>0 \text { and } d(Q(E), \delta q(E))>0
$$

for sufficiently small $b$. Choosing $b \neq 0$ and sufficiently small so that these several 
conditions hold we see that $Q(z)$ is univalent in $E$, has degree $n+1$ and $p(z) \subset Q(z)$ in $E$. Moreover, $m_{Q}(1)>0$ and $d(Q(E), \delta q(E))>0$ so that the argument that just was applied to $p(z)$ and $q(z)$ can again be applied to $Q(z)$ and $q(z)$. Continuing in this manner we obtain the chain of univalent polynomials

$$
p(z) \subset p_{n+1}(z) \subset p_{n+2}(z) \subset \cdots \subset p_{k-1}(z) \subset q(z),
$$

where $p_{j}(z)$ has degree $j$. In fact, there is an infinite such chain between $p(z)$ and $q(z)$, but for our purposes we end with $p_{k-1}(z)$.

Now we return to the earlier chain

$$
p_{n_{1}}(z) \subset p_{n_{2}}(z) \subset p_{n_{3}}(z) \subset \ldots .
$$

If $n_{1} \neq 1$ set $p_{1}(z)=a_{0}+b_{1} z$ where $b_{1} \neq 0$ and is sufficiently small so the closed disk $\left|w-a_{0}\right| \leqq\left|b_{1}\right|$ is contained in $p_{n_{1}}(E)$. Between successive pairs of polynomials in this new chain interject appropriate polynomials, as just described. This produces the chain

$$
p_{1}(z) \subset p_{2}(z) \subset p_{3}(z) \subset \cdots,
$$

where $p_{n}(z)$ is univalent in $E$ and is a polynomial of degree $n$. Moreover, a subsequence of $\left\{p_{n}(z)\right\}$, namely $\left\{p_{n}(z)\right\}$, converges to $f(z)$ uniformly in compact subsets of $E$. The proof will be complete if we can show that if $\left\{\varepsilon_{j}\right\}$ is any sequence of positive numbers such that $\varepsilon_{j} \rightarrow 0$ then the interjected polynomials can be so chosen to fill out the chain

so that

$$
p_{n_{1}}(z) \subset p_{n_{2}}(z) \subset p_{n_{3}}(z) \subset \ldots
$$

$$
\left|p_{n}(z)-p_{n_{j}}(z)\right|<\varepsilon_{j}
$$

for $z$ in $E$ and for $n_{j}<n<n_{j+1}, j=1,2, \ldots$

In order to do this consider the previous argument where $p(z)$ and $q(z)$ are successive pairs in the sequence $\left\{p_{n_{j}}(z)\right\}$ and have degrees $n$ and $k$, respectively, with $n<k$. That argument yielded real numbers $R_{\mu}$ and $\delta_{\mu}, \mu=n+1, \ldots, k-1$, such that $R_{\mu}>1, \delta_{\mu}>0$ and successively defining $p_{\mu}(z)$ by

$$
p_{\mu+1}(z)=p_{\mu}\left(\rho_{\mu} z\right)+b_{\mu+1} z^{\mu+1}
$$

the chain between $p(z)$ and $q(z)$ results, where $\rho_{\mu}$ and $b_{\mu}$ are restricted only by the conditions $1<\rho_{\mu}<R_{\mu}$ and $0<\left|b_{\mu}\right|<\delta_{\mu}$. The recursive definitions lead to the formula

$$
\begin{aligned}
p_{\mu}(z)= & p_{n}\left(\rho_{n} \rho_{n+1} \cdots \rho_{\mu-1} z\right)+b_{n+1}\left(\rho_{n+1} \rho_{n+2} \cdots \rho_{\mu-1}\right)^{n+1} z^{n+1} \\
& +b_{n+2}\left(\rho_{n+2} \rho_{n+3} \cdots \rho_{\mu-1}\right)^{n+2} z^{n+2}+\cdots+b_{\mu-1} \rho_{\mu-1}^{\mu-1} z^{\mu-1}+b_{\mu} z^{\mu} .
\end{aligned}
$$

Let $\varepsilon$ be any given positive number. Since $p_{n}(z)$ is uniformly continuous in $E$ there exist numbers $\rho_{\mu}$ such that $1<\rho_{\mu}<R_{\mu}$ and the product $\rho_{n+1} \rho_{n+2} \cdots \rho_{k-1}$ is so close to 1 that

$$
\left|p_{n}(z)-p_{n}\left(\rho_{n} \rho_{n+1} \cdots \rho_{\mu-1} z\right)\right|<\varepsilon / 2
$$


for $z$ in $E, \mu=n+1, \ldots, k-1$. Let such a choice of $\rho_{\mu}$ be made. From (1) it follows that if $z \in E$ then

$$
\begin{aligned}
\left|p_{\mu}(z)-p_{n}\left(\rho_{n} \rho_{n+1} \cdots \rho_{\mu-1} z\right)\right| \leqq & b_{n+1}\left|\left(\rho_{n+1} \cdots \rho_{\mu-1}\right)^{n+1}+\right| b_{n+2} \mid\left(\rho_{n+2} \cdots \rho_{\mu-1}\right)^{n+2} \\
& +\cdots+\left|b_{\mu-1}\right| \rho_{\mu-1}^{\mu-1}+\left|b_{\mu}\right| \\
& \leqq\left(\rho_{n+1} \rho_{n+2} \cdots \rho_{k-1}\right)^{n+1}\left\{\left|b_{n+1}\right|+\left|b_{n+2}\right|+\cdots+\left|b_{\mu}\right|\right\} \\
& \leqq\left(\rho_{n+1} \rho_{n+2} \cdots \rho_{k-1}\right)^{n+1}\left\{\left|b_{n+1}\right|+\left|b_{n+2}\right|+\cdots+\left|b_{k-1}\right|\right\} .
\end{aligned}
$$

Now choose the numbers $b_{\mu}, \mu=n+1, \ldots, k-1$, so that $0<\left|b_{\mu}\right|<\delta_{\mu}$ and the last expression in the previous line does not exceed $\varepsilon / 2$. This produces an appropriate chain

$$
p(z) \subset p_{n+1}(z) \subset \ldots \subset p_{k-1}(z) \subset q(z)
$$

satisfying the additional condition

$$
\left|p_{\mu}(z)-p_{n}(z)\right|<\varepsilon
$$

for $z$ in $E, \mu=n+1, \ldots, k-1$. This argument applied to $p_{n_{j}}(z)$ and $p_{n_{j+1}}(z)$, with $\varepsilon=\varepsilon_{j}$, yields the desired conclusion that $p_{n}(z) \rightarrow f(z)$ as $n \rightarrow \infty$ uniformly in compact subsets of $E$. This completes the proof in the case $f(z)$ is not a polynomial.

The case when $f(z)$ is a univalent polynomial can be treated in an even simpler way. Suppose that $f(z)=\sum_{k=0}^{N} a_{k} z^{k}, a_{N} \neq 0$, is univalent in $E$. Let $\left\{r_{j}\right\}$ be a sequence of real numbers such that $0<r_{1}<r_{2}<r_{3}<\cdots$ and $r_{j} \rightarrow 1$ and set $f_{j}(z)=f\left(r_{j} z\right)$. Choose $b_{1} \neq 0$ so small that $p_{1}(z)=a_{0}+b_{1} z$ is subordinate to $f_{1}(z)$ say by demanding the disk $\left|w-a_{0}\right| \leqq\left|b_{1}\right|$ is covered by $f_{1}(E)$. The relation between $p_{1}(z)$ and $p_{N}(z)$ $=f_{1}(z)$ implies the existence of polynomials $p_{n}(z), n=2, \ldots, N-1$ univalent in $E$ such that $p_{n}(z)$ has degree $n$ and

$$
p_{1}(z) \subset p_{2}(z) \subset p_{3}(z) \subset \cdots \subset p_{N-1}(z) \subset p_{N}(z),
$$

as was already shown in an earlier argument in this proof.

Next choose $b_{N+1} \neq 0$ so small that

$$
p_{N+1}(z)=f_{2}(z)+b_{N+1} z^{N+1}
$$

is univalent in $E$ and such that $d\left(p_{N}(E), \delta p_{N+1}(E)\right)>0$ and $d\left(p_{N+1}(E), \delta f_{3}(E)\right)>0$.

This is possible since $f_{2}(z)$ is univalent in a disk $|z|<R$ with $R>1, d\left(f_{1}(E), \delta f_{2}(E)\right.$ ) $>0$ and $d\left(f_{2}(E), \delta f(E)\right)>0$.

At the next step we select $b_{N+2} \neq 0$ so small that $p_{N+2}(z)=f_{3}(z)+b_{N+2} z^{N+1}$ is univalent in $E$ and such that $d\left(p_{N+1}(E), \delta p_{N+2}(E)\right)>0$ and $d\left(p_{N+2}(E), \delta f_{4}(E)\right)>0$.

This process can be continued to yield the chain

$$
p_{1}(z) \subset p_{2}(z) \subset p_{3}(z) \subset \cdots,
$$

where each $p_{n}(z)$ is a polynomial of degree $n$ univalent in $E$. Moreover, given any sequence of positive real numbers $\left\{\varepsilon_{j}\right\}$ such that $\varepsilon_{j} \rightarrow 0$ we can choose the numbers $b_{N+j}$ such that $\left|b_{N+j}\right|<\varepsilon_{j}$. This ensures that $p_{n}(z) \rightarrow f(z)$ uniformly in $E$. 
THEOREM 2. Suppose that $f(z)$ is analytic and univalent in $E$ and maps $E$ onto a convex domain. There exists a sequence $\left\{p_{n}(z)\right\}, n=1,2, \ldots$, such that $p_{n}(z)$ is a polynomial of degree $n$ that maps $E$ one-to-one onto a convex domain,

$$
p_{1}(z) \subset p_{2}(z) \subset p_{3}(z) \subset \cdots
$$

in $E$, and $p_{n}(z) \rightarrow f(z)$ uniformly in each compact subset of $E$.

Proof. A function $g(z)$ analytic in $E$ and satisfying $g^{\prime}(0) \neq 0$ maps $E$ one-to-one onto a convex domain if and only if

$$
\operatorname{Re}\left\{\frac{z g^{\prime \prime}(z)}{g^{\prime}(z)}+1\right\}>0
$$

for $z$ in $E$. Thus, if $0<r<1$ then such a function satisfies

$$
m_{g}^{*}(r)=\min _{|z| \leqq r} \operatorname{Re}\left\{\frac{z g^{\prime \prime}(z)}{g^{\prime}(z)}+1\right\}>0 .
$$

The proof of this theorem is essentially the same as that given for Theorem 1 where the role of $m_{g}(r)$ in that theorem is now replaced by $m_{g}^{*}(r)$. Again letting $s_{n}(z)$ be the $n$th partial sum of the power series for $f(z)$, we note that $s_{n}^{\prime}(z) \rightarrow f^{\prime}(z)$ and $s_{n}^{\prime \prime}(z) \rightarrow f^{\prime \prime}(z)$ uniformly in $|z| \leqq r, 0<r<1$. Consequently, there exists an integer $N=N(r)$ such that if $n \geqq N$ then

$$
m_{s_{n}}^{*}(r) \geqq m_{f}^{*}(r) / 2 .
$$

In particular, such functions $s_{n}(z)$ are univalent and convex in $|z| \leqq r$. Once this can be done it is clear that the other parts of the argument in Theorem 1 can be combined with this to yield (in the case $f(z)$ is not a polynomial) the polynomials $p_{n_{j}}(z)=s_{n_{j}}\left(r_{j} z\right)$ such that $0<r_{j}<1, r_{j} \rightarrow 1, p_{n_{j}}(z)$ is univalent and convex in $E$ and has degree $n_{j}$, and $n_{1}<n_{2}<n_{3}<\cdots$. Also,

in $E$,

$$
p_{n_{1}}(z) \subset p_{n_{2}}(z) \subset p_{n_{3}}(z) \subset \ldots
$$

and

$$
m_{p_{n_{j}}}^{*}(1) \geqq m_{f}^{*}\left(r_{j}\right) / 2
$$

$$
d\left(D_{n_{j}}\left(r_{j}\right), \delta D\left(r_{j+1}\right)\right)>0 .
$$

In order to interject convex univalent polynomials between two polynomials $p(z)$ and $q(z)$ which are consecutive in the sequence $\left\{p_{n_{j}}(z)\right\}$ first notice that $m_{p}^{*}(1)>0$ and therefore $p(z)$ is univalent and convex in $|z|<R$ for some $R>1$. Setting $P(z)$ $=p(\rho z)$ for $1<\rho<R$ we see that $m_{\rho}^{*}(1)>0$, and, consequently, if

$$
Q(z)=P(z)+b z^{n+1}
$$

then

$$
m_{Q}^{*}(1) \geqq \frac{1}{2} m_{P}^{*}(1)>0
$$

for all sufficiently small values of $b$. This implies that $Q(z)$ is univalent and convex 
in $E$. All the other arguments of Theorem 1 are applicable. In particular, at this point the relations between $p(z)$ and $q(z)$ now hold between $Q(z)$ and $q(z)$ so that the argument may be repeated. This produces the appropriate chain

$$
p_{1}(z) \subset p_{2}(z) \subset p_{3}(z) \subset \cdots,
$$

and, as before, a more careful construction of the interjected polynomials shows that such polynomials can be chosen which also satisfy $p_{n}(z) \rightarrow f(z)$ uniformly in compact subsets of $E$.

This outlines the proof when $f(z)$ is not a polynomial, and an argument like that given at the end of Theorem 1 takes care of the case when $f(z)$ is a polynomial.

REMARKS. 1. The method used to prove Theorems 1 and 2 also applies if $f(z)$ maps $E$ one-to-one onto a domain starlike with respect to the point $f(0)$. This yields univalent polynomials $\left\{p_{n}(z)\right\}$ which are starlike with respect to $f(0)$. One simply needs to note that a function $g(z)$ analytic in $E$ and satisfying $g^{\prime}(0) \neq 0$ is univalent and starlike (with respect to $g(0)$ ) in $E$ providing

$$
\operatorname{Re}\left\{\frac{z g^{\prime}(z)}{g(z)-g(0)}\right\}>0
$$

The role of $m_{g}(r)$ in Theorem 1 is now replaced by

$$
\min _{|z| \leqq r} \operatorname{Re}\left\{\frac{z g^{\prime}(z)}{g(z)-g(0)}\right\}
$$

One can readily state suitable general conditions on a given function $f(z)$ analytic and univalent in $E$ in order that the ideas of Theorem 1 yield a proof of the existence of such polynomials which additionally have the given property of $f(z)$. One such general statement is the following.

Let $w=F\left(u_{1}, u_{2}, \ldots, u_{n}\right)$ be defined for certain complex $n$-tuples $\left(u_{1}, u_{2}, \ldots, u_{n}\right)$. Then $f(z)$ has a certain "given property" if

$$
w=G(z)=F\left(z, f(z), f^{\prime}(z), \ldots, f^{(n-1)}(z)\right)
$$

is a nonconstant analytic function in $E$ and if the values of $G(z)$ are assumed to lie in a given open set. This includes as special cases starlike and convex (univalent) functions; namely, the value region for $G(z)$ in each case is $\operatorname{Re} w>0$ and the functions $w=F\left(u_{1}, \ldots, u_{n}\right)$ are

$$
w=u_{1} u_{3} /\left(u_{2}-f(0)\right) \text { and } \quad w=u_{1} u_{4} / u_{3}+1,
$$

respectively. This idea also can be expressed if $G(z)$ is replaced by a function of several variables. In particular, this would include the case of all univalent functions (Theorem 1). There the value region is $w \neq 0$ for the function $\Delta_{f}(u, v)$.

Finally, we mention one more example of this type of approximation, namely for the case $f(z)$ is close-to-convex. Such functions are defined by the conditions that they be analytic in $E$ and that there exist an analytic, univalent and convex 
function $g(z)$ such that $\operatorname{Re}\left\{f^{\prime}(z) / g^{\prime}(z)\right\}>0$ in $E$. Such functions $f(z)$ are univalent in $E$ [4]. If $0<r<1$ then $\left|g^{\prime}(z)\right| \geqq m_{g}(r)$ and, consequently,

$$
\left|\frac{f^{\prime}(z)}{g^{\prime}(z)}-\frac{s_{n}^{\prime}(z)}{g^{\prime}(z)}\right| \leqq \frac{1}{m_{g}(r)}\left|f^{\prime}(z)-s_{n}^{\prime}(z)\right|
$$

for $|z| \leqq r$. This shows that

$$
s_{n}^{\prime}(z) / g^{\prime}(z) \rightarrow f^{\prime}(z) / g^{\prime}(z)
$$

uniformly in $|z| \leqq r$. Setting

$$
\tilde{m}_{f}(r)=\min _{|z| \leqq r} \operatorname{Re}\left\{\frac{f^{\prime}(z)}{g^{\prime}(z)}\right\}
$$

we see that $\tilde{m}_{f}(r)$ can play the role of $m_{f}(r)$ in the argument of Theorem 1 . This produces an appropriate chain of polynomials each of which is close-to-convex (relative to $g(z))$ in $E$.

2. The proof of Theorem 1 is very constructive and when combined with precise inequalities for univalent functions gives specific information on how well $p_{n}(z)$ approximates $f(z)$. What is sought is an estimate on

$$
\max _{|z| \leqq r}\left|f(z)-p_{n}(z)\right|
$$

in terms of $n$ and $r$. We may assume that $f^{\prime}(0)=1$, as otherwise the factor $\left|f^{\prime}(0)\right|$ will generally appear. It is known that $s_{n}(z)$ are univalent in

$$
|z|<1-6(\log n) / n
$$

for $n \geqq 17$ [5], and, in fact, this can be slightly improved using the more recent result, $m_{f}(r) \geqq\left(1-r^{2}\right) / r^{2}$ (see [2, p. 120] or [3]). The quantity $d(r)$ can also be estimated from below in a fairly simple fashion to get

$$
d(r) \geqq \frac{1}{4}((1-r) /(1+r))^{2} .
$$

One only needs consider the interplay of these numbers $1-6(\log n) / n$ and $\frac{1}{4}((1-r) /(1+r))^{2}$, noticing that the interjected polynomials bear no significance as the sequence $\left\{\varepsilon_{j}\right\}$ in the proof is arbitrary. One finally applies these relations between $r_{j}$ and $n_{j}$ into the proof of Lemma 1 along with the estimate $\left|a_{k}\right|<e k$. A similar procedure is possible for other classes of univalent functions. In particular, use could be made of the results in [11, pp. 404-408].

\section{REFERENCES}

1. A. Bielecki and Z. Lewandowski, Sur certaines familles des fonctions $\alpha$-étoilées, Ann. Univ. Mariae Curie-Skłodowska Sect. A. 15 (1961), 45-55. MR 26 \#315.

2. G. M. Golusin, Geometrische Funktionentheorie, VEB Deutscher Verlag, Berlin, 1957. MR 19, 735.

3. J. A. Jenkins, On an inequality of Golusin, Amer. J. Math. 73 (1951), 181-185. MR 12, 816.

4. W. Kaplan, Close-to-convex schlicht functions, Michigan Math. J. 1 (1952), 169-185. MR 14, 966. 
5. V. Levin, Bemerkung zu den schlichten abbildungen des Einheitkreises, Jber. Deutsch. Math. Verein. 42 (1932), 68-70.

6. T. H. MacGregor, Certain integrals of univalent and convex functions, Math. Z. 103 (1968), 48-54. MR 36 \#5324.

7. C. Pommerenke, On sequences of subordinate functions, Michigan Math. J. 7 (1960), 181-185. MR 27 \#1598.

8. - Über die Subordination analytischer Funktionen, J. Reine Angew. Math. 218 (1965), 159-173. MR 31 \#4900.

9. - On the Loewner differential equation, Michigan Math. J. 13 (1966), 435-443. MR 34 \#6064.

10. G. Pólya and I. J. Schoenberg, Remarks on de la Vallée Poussin means and convex conformal maps of the circle, Pacific J. Math. 8 (1958), 295-334. MR 20 \#7181.

11. M. S. Robertson, On the theory of univalent functions, Ann. of Math. (2) 37 (1936), 374-408.

12. G. Szegö, Zur Theorie der schlichten abbildungen, Math. Ann. 100 (1928), 188-211.

State University OF New York,

Albany, New York 